\title{
Development of a Healthy Lifestyle Module to Improve Mental Health in Adolescent Students: Protocol of a Randomized Controlled Trial
}

\author{
Esra Tajik*1, Nasrin Omidvar ${ }^{2}$, Mohammadreza Mohammadi ${ }^{3}$ and Behnam Rashidkhani ${ }^{4}$ \\ +Psychiatry and psychology research center, Tehran University of Medical Sciences and Department of Community Nutrition, \\ National Nutrition and Food Technology Research Institute, Faculty of Nutrition and Food Technology, Shahid Beheshti \\ University of Medical Science, Iran
}

${ }^{2}$ Department of Community Nutrition, National Nutrition and Food Technology Research Institute, Faculty of Nutrition and Food Technology, Shahid Beheshti University of Medical Science, Iran

${ }^{3}$ Psychiatry and psychology research center, Tehran University of Medical Sciences

${ }^{4}$ Department of Community Nutrition, Faculty of Nutrition and Food Technology, Shahid Beheshti University of Medical Science, Tehran, Iran

*Corresponding author: Esra Tajik, Psychiatry and psychology research center, Tehran University of Medical Sciences and Department of Community Nutrition, National Nutrition and Food Technology Research Institute, Faculty of Nutrition and Food

Technology, Shahid Beheshti University of Medical Science, Iran

\section{ARTICLE INFO}

Received: 蔧 May 15, 2021

Published: 蔧 May 24, 2021

Citation: Esra Tajik, Nasrin Omidvar, Mohammadreza Mohammadi, Behnam Rashidkhani. Development of a Healthy Lifestyle Module to Improve Mental Health in Adolescent Students: Protocol of a Randomized Controlled Trial. Biomed J Sci \& Tech Res 36(1)-2021. BJSTR. MS.ID.005787.

\begin{abstract}
The current study aimed to determine if a lifestyle-based intervention program could improve the mental health of adolescent students. This study is a randomized controlled trial in eleven sessions among 370 adolescents with no severe score of general health questionnaire. Primary outcome assessment at pre-, post- and three-month followup tests includes general health score and the secondary outcomes include changes in eating habit, physical activity and stress management skills using healthy lifestyle questionnaire, eating behavior, daily activity, coping inventory for stressful situations and general health questionnaire. The findings of the current study will be used as a starting point of incessant, regular lifestyle education for adolescents, and for researchers and educators to improve the health and well-being globally.
\end{abstract}

Keywords: Healthy Lifestyle; Healthy Eating; Mental Health; Depression; Anxiety; Stress

\section{Background}

Adolescence is a critical transitional period of life, which is characterized by critical and serious physical and psychological changes [1,2]. According to the United Nations Children's Fund [3], there are 1.2 billion adolescents (16\% of the world's population) in the world. The Iranian population with an average annual growth rate of $1.07 \%$ increased to over 81 million by 2018 , there were then 12.3 million adolescents, about $15 \%$ of the country's population [4]. Lifestyle diseases such as metabolic syndrome, cancer, and mental diseases are diseases of civilization and the main cause of death in the world [5-8]. Among the causes of lifestyle diseases and poor mental health, factors such as unhealthy eating habits, low physical activity, coping weakness, and chronic psychological stress are some of the major ones [9,10,11]. Many of these critical conditions in adulthood, such as lifestyle diseases, some other chronic diseases, unhealthy eating habits and low physical activity are rooted in the adolescence and can lead to illness or premature death later in life $[12,13]$. Lifestyle recommendations are also associated with mental health in children and adolescents [14]. 
Emphasizing lifestyle recommendations such as diet, physical activity, sedentary behavior and sleep in children and adolescents may reduce the future burden of mental illness [14]. Globally, the prevalence of mental health problem is $10-20 \%$ in children and adolescents [15]. In the developing countries, $7.9 \%$ to $15.7 \%$ of adolescents suffer from mental disorders $[15,16,17]$.

The rise in the number of adolescents with mental disorders has become a major concern over the recent decades $[18,19]$. As a result, there are needs for not only psychotherapeutic and pharmacological treatments, but also a variety of special preventive services such as educational interventions [20-22].

\section{Intervention}

Unhealthy lifestyle factors such as unhealthy eating, physical inactivity and poor stress management skills can contribute to mental health disorders, like depression, stress and anxiety [23]. Mental health disorders affect many adolescents [24]. The current research states the potential of lifestyle improvement to improve mental health status. Generally, despite the presence of effective approaches to reduce mental disorders, they are highly neglected [25]. Evidence in developed and developing countries has shown the effect of healthy lifestyle interventions on improving mental health status $[26,27]$. Therefore, there is a need to design and implement lifestyle change interventions targeted at adolescents to improve their mental and general health. In this regard, psychosocial and nutritional status and their determinants need to be evaluated and considered before planning or starting any strategy involving adolescents. Overall, the three top components of healthy lifestyle include healthy diet, physical activity and stress management which have often been neglected [28-30]. In Iran, healthy lifestyle education is very new, and there is a lack of systematic lifestyle training for adolescents. The lack of lifestyle-based education places Iranian adolescents at a higher risk of behavioral and mental problems such as stress, anxiety and depression [31]. Based on our knowledge, the present study is one of the first attempts in the country to implement a healthy lifestyle education intervention aim to improve mental health. Findings of the current research are hoped to be used by health care professionals and practitioners, policymakers and teachers to promote healthier lifestyle by not only psychological parameters but nutrition and physical activities in the adolescent community.

\section{Theoretical Framework}

The lifestyle behavior needs to be fully explicated within a theoretical context. Few studies have applied behavioral change theories on children and/or adolescents for promoting a healthy lifestyle [32,33]. Among the available theories, those with fewer factors and containing motivational factor are considered as more appropriate models for adolescents [34,35]. The InformationMotivation-Behavioral skill (IMB) model moves beyond the Health Belief Model and Social Cognitive Theory by suggesting that information, motivation, and behavioral skills are the primary individual level determinants of adopting preventive behaviors [36]. The IMB model has been tested for the prediction of healthy lifestyle behaviors, physical activity and dietary intake in adolescents [32]. Based on the Information-Motivation-Behavioral skill model, people who are well informed, motivated to act and practice, and have behavioral skills will maintain health-promoting behaviors and experience proper health outcomes [34-36]. The present study using IMB model has developed an interventional program to improve mental health in adolescents. Within the framework of the model, improvement of knowledge, attitude and behavior for healthy eating, physical activity and stress management skills were emphasized [37]. The theoretical framework of study based on IMB model.

\section{Design and Method Strength}

\section{Aim and Hypotheses}

The current study aims to develop a healthy lifestyle (healthy eating, physical activity and stress management skills) education module to improve mental health among student adolescents in Tehran, Iran.

The main research hypotheses are as follows:

a) There is a significant difference in scores of knowledge, attitude and behavior for healthy diet, physical activity and stress management skills among adolescents in the intervention group compared to control group.

b) There is a significant difference in general health score between the intervention and control groups at the end of the intervention.

\section{Study Design}

The present study is a single blinded randomized controlled trial (RCT) using a multi-stage sampling method. This RCT included a pre-, post- and three-month follow-up test presents schematic overview of the study steps.

\section{Participants}

The target population of the study is girl and boy students of high schools in three different socioeconomic districts of Tehran city, including deprived, mid-level and prosperous districts. Students aged 13-15 years who are able to write and read and are from public schools will be included to the study. Students will be excluded from the study if they have severe emotional distress (based on general health questionnaire) or those with diagnosed psychiatric illnesses; and also, students who refuse to fill the consent form

\section{Sample Size Calculation}

Calculation of sample size for the current RCT is based on Aday and Cornelius [39], where $\mathrm{n}$ is sample size in each group; Z1- $\alpha$ and 
Z1- $\beta$ equal 1.96 and 0.84, respectively. P1 (19.1\%) and P2 (3.2\%) according to previous study by Eslami et al. [40] are proportion of stress status between intervention and control groups after an interventional study. After calculating the " $n$ ", adding $20 \%$ for non-responding and enhancing by a coefficient of 1.2 for estimated design effect, the final sample size is 117 for each intervention and control groups.

\section{Sampling Technique and Recruitment}

A Multi-Stage Sampling Frame is Applied Through the Following Steps: First, the list of districts of Tehran was taken from formal documents of the ministry of education in which districts are categorized based on their socioeconomic status into deprived, mid-level and prosperous. In the second step, 12 schools from the 3 levels of districts were randomly selected and in each selected district 2 boys' schools and 2 girls' schools were listed. Then schools were equally allocated into intervention and control groups based on the feasibility and permissiveness. Finally, a screening study will be conducted among 1200 students based on General Health Questionnaire (GHQ) and after consulting with the school principal and the teachers, one class in each school will be randomly selected to participate to the study. Respecting ethical issues and avoiding any inconvenience, students with the exclusion criteria will not be taken out of classes. Therefore, all the students in each class will take part in the study and in total almost 370 participants will fill the questionnaires at the pre-intervention stage.

\section{Developing and Pilot Testing the Intervention}

The educational package based on the IMB model was developed in Persian, comprising stress management, physical activity and healthy diet modules (11 sessions). The main teaching strategies include group discussion, debate, videos (animation), research book, health talk, musical exercise, relaxation activities, games and competition. The educational package was developed through highlighting the strength and minimizing the weakness of current existing modules in some other countries, including
US [41-43], Iran [44], Malaysia [45-47], Hongkong [48] and etc. In the process of developing, the package was revised by a panel of health professionals (psychiatry, psychologist, and nutritionist) from Shahid Beheshti Medical science University (SBMU), Tehran University of Medical Sciences (TUMS), Ministry of Health, Ministry of Education and National Elites Foundation through a series of round tables.

Content Validity: The content of developed package was evaluated in terms of acceptability of the module such as title of the module, title of each session, appropriate level of content, reading level, design, graphic, activities (games and competitions), time and etc. Each item was assessed by health professionals, teachers and adolescent students ranging from poor (0) to very good (4) covering all aspects of the module.

Face Validity: The developed educational module was tested in a high school which will not be included in the main study. It was tested among 35 students for clarity of meaning, language, the flow of contents and the time needed for presenting. Each student signed a consent form before the beginning of educational sessions. In line with piloting the module, the pre-testing of the questionnaire set was conducted.

The final draft of healthy lifestyle educational module with 11 sessions (Table 1) was named as "TAVANA" which is the acronym of four words in Persian language. It stands for TA "Taghzieh salem" (healthy eating), V "Varzesh" (exercise), A "Aramesh: (calmness), NA "Nadaram stress" (no stress). The educational module is comprised of one session for "Introduction and Ice-breaking" and 10 sessions to cover the 3 main domains of healthy lifestyle, including stress management skills (knowing self, knowing stress situations, managing stressful situations, coping strategies and relaxation activity), physical activity (musical and stretching exercises and some educational games such as toxic ball) and healthy diet (balanced diet, my healthy plate, importance of main meals, fruits and vegetables).

Table 1: The content, activities and learning outcomes of the educational sessions.

\begin{tabular}{|c|c|c|c|}
\hline \multicolumn{2}{|r|}{ Session Title } & Activities & Learning Outcome \\
\hline 1 & Introduction and Icebreaking & $\begin{array}{l}\text { Welcoming and briefing the goals of the } \\
\text { intervention. }\end{array}$ & Familiarity with research team and feeling relax. \\
\hline 2 & Who am I? & $\begin{array}{l}\text { - Brief speech to deliver information. } \\
\text { - Watching video to visualize information and } \\
\text { increasing motivation. }\end{array}$ & $\begin{array}{l}\text { - Self-knowing as adolescent. } \\
\text { - Make challenges to know their weaknesses and } \\
\text { strengths. }\end{array}$ \\
\hline 3 & Healthy lifestyle & $\begin{array}{l}\text { - Brief speech to deliver information } \\
\text { - Watching attractive slides to visualize information } \\
\text { and increasing motivation. }\end{array}$ & $\begin{array}{l}\text { Increasing knowledge on the importance of healthy } \\
\text { lifestyle components. }\end{array}$ \\
\hline 4 & Relaxation activity & $\begin{array}{l}\text { - Brief talk about the Importance of relaxation } \\
\text { activity to reduce stress. } \\
\text { - Live demo of relaxation activities. }\end{array}$ & $\begin{array}{l}\text { Increasing knowledge on the importance of relaxation } \\
\text { activity to reduce stress. }\end{array}$ \\
\hline 5 & What is stress? & $\begin{array}{l}\text { - Brief speech to deliver information. } \\
\text { - Watching video to visualize information and } \\
\text { increasing motivation. }\end{array}$ & $\begin{array}{l}\text { Increasing knowledge on stress, stress situation and the } \\
\text { importance of coping. }\end{array}$ \\
\hline
\end{tabular}




\begin{tabular}{|c|c|l|l|}
\hline 6 & Managing stress wisely! & $\begin{array}{l}\text { - Team-working competitions and games. } \\
\text { - Offering different coping strategies. }\end{array}$ & Learning how to control stress in unsuitable situations. \\
\hline 7 & Live, Camera, Action! & \multicolumn{1}{|c|}{ Role playing for coping strategies. } & Coping strategies, moral values in different situations. \\
\hline 8 & Be physically active! & $\begin{array}{l}\text { - Brief speech to deliver information. } \\
\text { - Some attractive team-working games. }\end{array}$ & $\begin{array}{l}\text { Increasing knowledge on the importance of physical } \\
\text { activity and disadvantages of sedentary lifestyle. }\end{array}$ \\
\hline 9 & Balanced diet & $\begin{array}{l}\text { - Brief speech to deliver information. } \\
\text { - Some attractive competition and games using food } \\
\text { cards and puzzles. }\end{array}$ & $\begin{array}{l}\text { - Increasing knowledge on the importance of healthy diet, } \\
\text { food groups and food pyramid. } \\
\text { - Reducing intake of sweet and fast food. }\end{array}$ \\
\hline 10 & 3 Main meals & $\begin{array}{l}\text { - Brief speech to deliver information. } \\
\text { - Some attractive competition and games using food } \\
\text { cards. } \\
\text { - Watching video to visualize information and } \\
\text { increasing motivation. }\end{array}$ & $\begin{array}{l}\text { - Increasing knowledge on the importance of breakfast } \\
\text { and other meals. } \\
\text { - Reducing missing main meals. }\end{array}$ \\
\hline \multirow{2}{*}{11} & Fruits and Vegetables & $\begin{array}{l}\text { - Brief speech to deliver information. } \\
- \text { Team-working competition to make healthy snacks. }\end{array}$ & $\begin{array}{l}\text { - Increasing knowledge on the importance of fruit and } \\
\text { vegetable. } \\
\text { - Preparing healthy snacks for school time or at home. }\end{array}$ \\
\hline
\end{tabular}

The sessions will be held once per week for one hour in each intervention school. Each session starts with relaxation activities and finishes by Q\&A and musical exercise. Researcher will make the rules to control the educational sessions and their discipline. The rules of attending classes will be reviewed for students at the beginning of each session. The required materials and stationeries will be provided and checked by research team before each session. No intervention will be conducted in the control group during data collection. The control group will receive the educational instruments and materials at the end of the study.

\section{Measurements and Outcomes}

Data will be collected by six, self-administered questionnaires to measure demographics, knowledge, attitude and practice for healthy lifestyle, healthy diet, physical activity and stress management skills including relaxation activities and coping. The questionnaires were developed by the expert panel in Shahid Beheshti Medical Sciences University (SBMU) and Tehran University of Medical Sciences (TUMS). The questionnaire also was adapted from the previous study on healthy lifestyle by Tajik et al. [49] which was conducted among adolescents. The process of review and revising of the questionnaires was done considering the module content. Reliability and validity of the questionnaires were assessed through evaluating their face and content validity, as well as internal consistency.

A. The Study Questionnaires are as Follows:

Demographic Questionnaire: age, gender, ethnicity, household size, monthly household income, parental educational level, parental occupation and the menarche age of female students.

Knowledge Questionnaire: Students' knowledge will be assessed by 34-item, self-administered questionnaire (including sub-scales on healthy lifestyle, healthy diet, physical activity, selfidentification, relaxation activity and coping). The questionnaire was developed and modified based on previous studies [50-57]. The knowledge questionnaire consisted of five multiple-choice questions that the fifth is "I do not know" and rest of four choices is facts which only one of them is a true answer.

Attitude Questionnaire: The questionnaire on attitude included 31 items (on healthy lifestyle, healthy diet, physical activity, self-identification, relaxation activity and coping), with 5-point scale response options, ranging from 'strongly agree (1 point)' to 'strongly disagree (5 points)'. It was developed based on previous studies [49-62] by the study team.

Eating Behavior Questionnaire: The Eating behavior questionnaire was developed and modified based on two preexisting questionnaires: Eating Behaviors Questionnaire (EBQ) (62) and food habits [53]. This questionnaire assessed frequency of meal consumption and snacking between meals; frequency of eating outside, fast foods, sweet foods and beverages, and frequency of food groups (fruits, vegetables, dairy and fish), type of snacks consumption, use and type of dietary supplement. The questionnaire according to the goal of intervention was modified adding questions related to 'frequency of food groups' Frequencies ranged from every day (score 7) to less than once a month or never (score 0). Internal consistency for the current study is 0.791 . Skipping at least one meal per day will be considered as skipping meal and snacking.

Daily Activity Questionnaire: This questionnaire which was developed and validated by Kelishadi et al. [63], included 9levels of physical activity, ranging from inactivity (0.9 METs) to vigorous activity ( $>6$ METs). The cronbach's alpha value of the scale was 0.87 , which indicates acceptable internal consistency. 
Coping Inventory for Stressful Situations (CISS): CISS is developed by Endler and Parker [64] and focused on identification and comparison of basic coping strategies used by different individuals across different types of stressful situations [64]. The CISS is a 48-item scale that measures three basic coping strategies: Task-Oriented, Emotion-Oriented, and Avoidance (distraction and social items), with 16 items per scale. These 5 -point Likert scales are scored from 1 (not at all) to 5 (very much). The higher summing scores indicate a greater use of that particular coping strategy. The Cronbach's alpha reported for the scale and its sub-scales range from 0.72 to 0.92 [64]. The Persian version of the CISS was validated [65] with the Cronbach's alpha of 0.70 to 0.86 for coping scales.

General Health Questionnaire-28 (GHQ-28): The GHQ-28 was developed and validated by Goldberg and Hillier [66] to detect the risk of developing psychiatric disorder or to measure emotional distress. The questionnaire is consisted of 28 questions in 4 Likert scale from 0 (not at all) to 3 (much more than usual). The scores above 22 indicate disorder symptoms. The Cronbach's alpha coefficient of the Persian version of GHQ is 0.90 [67].

Relaxation Activity: This questionnaire was developed based on the frequency of practicing relaxation activity techniques for muscle relaxation activity and deep breathing activity according to the literature review $[49,68,69]$. Two questions on the duration and frequency of doing relaxation will assess the relaxation activities. The frequency of relaxation activities was including: none, 1 time, 2 or 3 times, 4 or 5 times, 6 or 7 times. The scores was ranging from 0 (none) to 4 ( 6 or 7 times). Total relaxation activity practice per week was calculated as the sum of the two individual behaviors per

Table 2: The primary and secondary outcomes of the study. week.

\section{Data Collection Procedure}

Data will be collected using a set of structured, pre-tested and self-administrative questionnaires in Persian language. After identifying appropriate schools, the agreements will be done by telephone and face to face meeting with the schools' principals. The principals of the 12 selected schools were informed about the objectives of the study and how the process of data collection would be. After obtaining the approval from the school principal, participants and their parents will be given consent forms one week before data collection and they will be asked to return back the signed consent forms. The principal and teachers of the school will inform the students of the data collection process. After making an appointment for baseline data collection, the self-administered questionnaires will be handed to participants to be filled out in the classes. First, research team will explain all questionnaires step by step to participants and will ask them to complete the questionnaires truly. After filling the questionnaires, the anthropometry measurements, including body weight and height will be measured using calibrated tools by research team.

The educational sessions will be commenced in 5 months (September-January). A baseline data will be collected a week prior to the intervention and subsequently an immediate post-test and a three-month follow-up test will be carried out for both groups (control and intervention) using the same questionnaire set.

Expected Outcomes: The expected study outcomes including the primary and secondary outcomes which will be implemented in sequence are presented in Table 2.

\begin{tabular}{|c|c|c|}
\hline Outcome Level & Outcomes & \\
\hline \multirow{5}{*}{ Primary } & - Increase in the mean score of knowledge on & $\begin{array}{ll}\cdot & \text { Healthy diet } \\
\cdot & \text { Physical activity } \\
\text {. } & \text { Stress management }\end{array}$ \\
\hline & - Increase in the mean score of attitudes on & $\begin{array}{ll}\text { o } & \text { Healthy diet } \\
\text { o } & \text { Physical activity } \\
\text { o } & \text { Stress management }\end{array}$ \\
\hline & - Improve in healthy eating score & \\
\hline & - Improve in physical activity level & \\
\hline & $\begin{array}{l}\text { - Improve in stress management skills (coping stress and } \\
\text { relaxation activities) }\end{array}$ & \\
\hline Secondary & Reduction in the mean score of GHQ & \\
\hline
\end{tabular}

\section{Data Analysis}

Data will be analyzed using the SPSS software version 21.0. Normality of data will be checked by the value of skewness/std. error less than 1.96, and histogram values. For data which are not normally distributed, a log transformation can be used. The alpha level at 0.05 will be taken as the level of statistical significance for all the variables in the analysis. Independent t-test will be done to determine the differences in the mean between intervention and control groups. For the nominal and ordinal variables, Chisquare test will be used to test the association between categorical dependent and independent variables. Two-way repeated measure 
ANOVA controlling for baseline socio-demographic data, will be performed to test whether there is significant difference in the means score between study groups at different times over the period of study and also for the effect of time (baseline, at 3 months and 6 months follow-up assessments) in each group.

\section{Discussion}

This paper presents the rationale and protocol for the healthy lifestyle intervention among adolescents. To the best our knowledge, this is an innovative intervention and the first randomized lifestyle change educational program, using three main domains of stress management, diet and physical activity. Other studies tried to introduce lifestyle to adolescents and/or adults conducting physical activity and healthy diet $[70,71]$. Conducting the trial in schools with different socio-economical levels is important to evaluate feasibility and effectiveness of the intervention. As far as some lifestyle skills may be improved in poverty and some in wealth [72,73]. Moreover, adolescents meet lots of crises in the specific period of life while have a bit skills to solve or manage them. Targeting adolescents that do not have enough information about themselves and their health is one of the crucial requirements for each country to have healthy society in future. Poor knowledge on lifestyle can lead to poor health and subsequently psychosocial problems and/or poor diet which are the major problems among adolescents especially in urban settings [74]. Although these issues have often been neglected, early intervention can prevent and improve emotional disturbances. Paying attention to factors related to adolescent mental health, lifestyle and dietary behavior is an urgent necessity. Improving mental health will require increasing knowledge, changing attitude and improving stress management skills, diet quality and physical activity $[43,75,76]$, as the components of healthy lifestyle $[77,78]$

Previous studies have mainly focused on coping strategies to deal with negative emotions such as stress, sadness, and anxiety $[79,80]$, while in the current study, positive approach to stress management is emphasized, including active coping, getting consultation from wise people, teachers and parents, role playing to find solutions for different difficulties, as well as relaxation activities. For instance, the coping strategies included in the module involved activities such as improving children's abilities, feelings, communications, and networking to help them cope more effectively with changes in life. And also each session will be continued by performing physical activity or exercise to improve mental health even in the presence of serious mental problems [81]. Finally, increasing knowledge and improving attitude and behaviour on healthy diet aims to reduce stress oxidative and boost mental health as well as reduce the risk of mental disorders. Through the proposed intervention, fruits, vegetables, whole brans, fish, and also dairy products and water are promoted as a mean to reduce stress. In line with this approach, O'Neil et al. [82] proposed a dietary intervention to improve mental health. Their protocol involved some dietary changes, including eating fruits, vegetables, whole grains, fish, olive oil, legumes and nuts. A study by Kulkarni et al. [83] showed that healthy eating was significant associated with mental health and unhealthy eating was associated with mental distress $(\mathrm{p}<0.05)$. Contrary, a descriptive study among middle-aged individuals stated that healthy diet had no significant associated with mental health [84].

However, although programs on improving healthy diets, physical activities and psychosocial wellbeing are being implemented among adolescents, comprehensive approaches on healthy lifestyle considering its main components in one package is still rare. Accordingly, the findings of this study will be useful for health educators, health promoters, and nutritionists who provide an opportunity to impress adolescents' requires such as coping, eating and physical activities. In addition, the educational program can be used for future interventions which are based on behavioral changes. The findings of this study will provide useful information for policy makers in this area to focus on interventional strategies that will address behavioral changes among adolescents to improve mental health.

\section{Timeline and The Current Status of the Trial}

The research study will be conducted over more than one year comprising preparation of the educational package, pilot study, and implementation of study, data analysis and writing the report. Permissions and recruitment of participants commenced in winter 2018.

\section{Ethics Approval and Consent to Participate}

The project got ethic approval from the ethical committee of faculty of nutritional sciences and food technology, Shahid Beheshti Medical University, coded IR.SBMU.nnftri.1395.199. In addition, the ministry of education of Iran, educational offices of 6 districts of Tehran city, principals of 12 secondary schools, teachers, students and their parents will be informed about the objectives of the study. Moreover, written consent forms will be signed by the students and their parents before beginning of data collection.

\section{Competing Interest}

There is no competing interest for authors.

\section{Acknowledgements}

We would like to express our sincere gratitude to managements and teachers of schools for their valuable cooperation.

\section{References}

1. Hindin MJ, Christiansen CS, Ferguson BJ (2013) Setting research priorities for adolescent sexual and reproductive health in low- and middle-income countries. Bull World Health Organ 91(1): 10-18.

2. Blakemore SJ (2012) Development of the social brain in adolescence. J R Soc Med 105(3): 111-116.

3. (2018) Adolescent's overview: UNICEF.

4. (2018) The statistics portal of Iran. 
5. Tabish SA (2017) Lifestyle Diseases: Consequences, Characteristics, Causes and Control. J Cardiol Curr Res 9(3): 00326.

6. (2010) Global status report on noncommunicable disease 2010. World Health Organization.

7. Parkin DM, Boyd L, Walker LC (2010) The fraction of cancer attributable to lifestyle and environmental factors in the UK. Br J Cancer 105(Suppl 2): S77-S81.

8. Kvaavik E, Batty GD, Ursin G, Huxley R, Gale CR (2010) Influence of individual and combined health behaviors on total and cause-specific mortality in men and women: The United Kingdom health and lifestyle survey. Arch Intern Med 170(8): 711-718.

9. Rao S, Shah N, Jawed N, Inam S, Shafique K (2015) Nutritional and lifestyle risk behaviors and their association with mental health and violence among Pakistani adolescents: results from the National Survey of 4583 individuals. BMC Public Health 15: 431.

10. Qidwai W, Ishaque S, Shah S, Rahim M (2010) Adolescent Lifestyle and Behaviour: A Survey from a Developing Country. PLoS One 5(9): e12914.

11. (2014) European Society of Lifestyle Medicine.

12. Waddington C, Sambo C (2015) Financing health care for adolescents: a necessary part of universal health coverage. Bull World Health Organ 93(1): 57-59.

13. Stevens S, Morral A (2013) Adolescent Substance Abuse Treatment in the United States. Routledge, New York, USA, $2^{\text {nd }}$ Ed.

14. Olivia K Loewen, Katerina Maximova, John P Ekwaru, Erin L Faught, Mark Asbridge, et al. (2019) Pediatrics 143(5): e20183307.

15. Kieling CH, Baker Henningham H, Belfer M, Conti G, Ertem I, et al. (2011) Child and adolescent mental health worldwide: evidence for action. The LANCET 378(9801): 1515-1525.

16. Jalali M, Pourahmadi E (2012) Prevalence of anxiety disorders among 10-14 years old children in Gorgan. European Psychiatry 27(Suppl 1): 1.

17. Zarafshan H, Mohammadi MR, Salmanian M (2015) Prevalence of Anxiety Disorders among Children and Adolescents in Iran: A Systematic Review. Iran J Psychiatry 10(1): 1-7.

18. Insel TR (2014) Mental disorders in childhood: Shifting the focus from behavioral symptoms to neurodevelopmental trajectories. JAMA 311(17): 1727-1728.

19. Whiteford HA, Degenhardt L, Rehm JT, Baxter AJ, Ferrari AJ, et al. (2013) Global burden of disease attributable to mental and substance use disorders: Findings from the Global Burden of Disease Study 2010. The Lancet 382(9904): 1575-1586.

20. Polanczyk G, Salum G, Sugaya L, Caye A, Rohde L (2015) Annual Research Review: A meta-analysis of the worldwide prevalence of mental disorders in children and adolescents. The Journal of Child Psychology and Psychiatry 56(3): 345-365.

21. Olfson M, Blanco C, Wang S, Laje G, Correll CU (2014) National trends in the mental health care of children, adolescents, and adults by office based physicians. JAMA Psychiatry 71(1): 81.

22. Atladottir HO, Gyllenberg D, Langridge A, Sandin S, Hansen SN, et al. (2014) The increasing prevalence of reported diagnoses of childhood psychiatric disorders: A descriptive multinational comparison. European Child \& Adolescent Psychiatry 24(2): 173-183.

23. Jacka FN (2015) Lifestyle factors in preventing mental health disorders: an interview with Felice Jacka. BMC Med 13(1): 264.

24. (2019) Adolescent mental health. World health Organization.

25. Shrivastava A, Desousa A (2016) Resilience: A psychobiological construct for psychiatric disorders. Indian J Psychiatry 58(1): 38-43.

26. Velten J, Lavallee KL, Scholten S, Meyer AH, Zhang X, et al. (2014) Lifestyle choices and mental health: a representative population survey. BMC Psychol 2: 58.
27. Tajik E, Latiffah AL, Hamidin A, Siti Nur'Asyura A, Chin YS, et al. (2016) Unhealthy diet practice and symptoms of stress and depression among adolescents in Pasir Gudang, Malaysia. Journal of Obesity Research and Clinical Practice 10(2): 114-123.

28. Jacka FN, Kremer PJ, Berk M, de Silva-Sanigorski AM, Moodie M, et al. (2011) A Prospective Study of Diet Quality and Mental Health in Adolescents. PLoS ONE 6(9): e24805.

29. Jacka FN, Kremer PJ, Leslie ER, Berk M, Patton GC, et al. (2010) Associations between diet quality and depressed mood in adolescents: results from the Australian Healthy Neighbourhoods Study. Australian and New Zealand Journal of Psychiatry 44(5): 435-442.

30. Melnyk BM, Jacobson D, Kelly S, O'Haver J, Small L, et al. (2009) Improving the Mental Health, Healthy Lifestyle Choices, and Physical Health of Hispanic Adolescents: A Randomized Controlled Pilot Study. Journal of School Health 79(12): 575-584.

31. Abbasalizad Farhangi M, Dehghan P, Jahangiry L (2018) Mental health problems in relation to eating behavior patterns, nutrient intakes and health related quality of life among Iranian female adolescents. PLoS One 13(4): e0195669.

32. Kelly S, Melnyk BM, Belyea M (2012) Predicting Physical Activity and Fruit and Vegetable Intake in Adolescents: A Test of the Information, Motivation, Behavioral Skills Model. Research in Nursing \& Health 35(2): 146-163.

33. Kelly S, Melnyk B (2008) Systematic Review of Multicomponent Interventions with Overweight Middle Adolescents: Implications for Clinical Practice and Future Research. Worldviews on Evidence-based Nursing 5(3): 113-135

34. Baranowski T, Cullen K, Baranowski J (1999) Psychosocial Correlates of Dietary Intake: Advancing Dietary Intervention. Annual Review of Nutrition 19: 17-40.

35. Gao J, Wang J, Zhu Y, Yu J (2013) Validation of an information-motivationbehavioral skills model of self-care among Chinese adults with type 2 diabetes. BMC Public Health 13: 100.

36. Fisher J, Fisher W, Bryan A, Misovich S (2002) Information-motivation behavioral skills model-based HIV risk behavior change intervention for innercity high school youth. Health Psychology 21(2): 177-186.

37. Fisher JD, Fisher WA, Amico KR, Harman JJ (2006) An informationmotivation-behavioral skills model of adherence to antiretroviral therapy. Health Psychol 25(4): 462-473.

38. Osborn CY, Amico KR, Fisher WA, Egede LE, Fisher JD (2010) An Information-Motivation-Behavioral Skills Analysis of Diet and Exercise Behavior in Puerto Ricans with Diabetes. J Health Psychol 15(8): 1201-1213.

39. Aday AL, Cornelius LJ (2006) Designing and conducting health surveys: a comprehensive guide. Published by Jossey-Bass. Foreword by Steven B. Cohen, $3^{\text {rd }}$ Ed.

40. Eslami AA, Rabiei L, Afzali SM, Hamidizadeh S, Masoudi R (2016) The Effectiveness of Assertiveness Training on the Levels of Stress, Anxiety, and Depression of High School Students. Iran Red Crescent Med J., 18(1): e21096.

41. Smith D, Horowitz M, Neelon M, Spezzano T, Lippitt N, et al. (2012) Healthalicious cooking. University of California, department of Nutrition.

42. (2005) Nutrition Education in Primary Schools: FAO.

43. Clarke G, Lewinsohn P, Hops H (1990) Leader's manual for adolescent groups. Adolescent coping with depression course. Center for health Research pp. 300.

44. (2013) Ministry of Health of Iran and Nutrition Improvement Office. Nutrition Training Module for the Health Team.

45. (2012) Ministry of Health of Malaysia. Module Ltihan Program Siswa Sihat (PROSIS) pp.155. 
46. (2011) Ministry of Health of Malaysia. Module Kesihatan Mental Remaja pp. 133.

47. Mohd nasir MT, Rokiah MY (2008) Module Gaya Hidup Sihat pp. 125.

48. The Hong Kong Polytechnic University, World Health Organization Collaborative Centre for Community Health Services. Integration of Adolescent Health and Development Domains into pre-service nursing curriculum, Module 6 Promoting Healthy Lifestyle Behaviours for Adolescents pp. 39.

49. Tajik E, Abd Latiff L, Yit Siew C, Awang H, Adznam SNA (2020) Designing and Validating a Questionnaire on Healthy Lifestyle to Reduce Depressive Symptoms among Adolescents. Iran J Psychiatry 15(1): 27-40.

50. Pelletier LG, Dion, Angelo SC, Slovinec D’Angelo M, Reid R (2004) Why do you regulate what you eat? Relationships between forms of regulation, eating behaviors, sustained dietary behavior change, and psychological adjustment. Motivation and Emotion 28(3): 245-277.

51. Morrow JR, Krzewinski Malone JA, Jackson AW, Bungum TJ, FitzGerald SJ (2004) American Adults' Knowledge of Exercise Recommendations. Research Quarterly for Exercise and Sport 75(3): 231-237.

52. Fielder CR (2008) Knowledge, Motivations, and Behaviors Regarding Eating a Healthy Diet and Physical Activity in Relation to Self-Esteem in College Students. Texas State University-San Marcos.

53. Turconi G, Celsa M, Rezzani C, Biino G, Sartirana MA, et al. (2003) Reliability of a dietary questionnaire on food habits,eating behaviour and nutritional knowledge of adolescents. European Journal of Clinical Nutrition 57(6): 753-763.

54. Stevens J, Cornell CE, Story M, French SA, Levin S, et al. (1999) Development of a questionnaire to assess knowledge, attitudes, and behaviors in American Indian children. Am J Clin Nutr 69(4): 773s-781s.

55. Meyer TJ, Miller ML, Metzger RL, Borkovec TD (1990) Development and validation of the Penn state worry questionnaire. Behavior Research Ther 28(6): 487-495.

56. Pearlin LI, Schooler C (1978) The structure of coping. National institute of mental health. Journal of health and social behavior 19(1): 2-21.

57. Fuqua JL, Gallaher PE, Unger JB, Trinidad DR, Sussman et al. (2012) Multiple Peer Group Self-Identification and Adolescent Tobacco Use. Subst Use Misuse 47(6): 757-766.

58. Garner DM, Olmsted MP, Bohr Y, Garfinkel PE (1982) The Eating Attitudes Test: Psychometric features and clinical correlates. Psychological Medicine 12(4): 871-878.

59. Kato Y, Iwanaga M, Roth R, Hamasaki T, Greimel E (2013) Psychometric Validation of the Motivation for Healthy Eating Scale (MHES). Psychology 4(2): 136-141.

60. Nåden D, Eriksson K (2004) Understanding the Importance of Values and Moral Attitudes in Nursing Carein Preserving Human Dignity. Nursing Science Quarterly 17(1): 86-91.

61. Kentsch M, Rodemerk U, Müller-Esch G, Schnoor U, Münzel T, et al. (2002) Emotional attitudes toward symptoms and inadequate coping strategies are major determinants of patient delay in acute myocardial infarction. Zeitschrift für Kardiologie 91(2): 147-155.

62. Chin YS, Mohd Nasir MT (2009) Eating Behaviors among Female Adolescents in Kuantan District, Pahang, Malaysia. Pakistan J Nutr 8(4): 425-432.

63. Kelishadi R, Rabie K, Khosravi A, Famori F, Sadeghi M, et al. (2001) Assessment of Physical Activity of Adolescents in Isfahan. J Shahrekord Univ Med Sci 3(2): 27-33.

64. Endler NS, Parker JD (1990) Coping Inventory for Stressful Situations (CISS): Manual. Toronto, ON, Canada: Multi-Health Systems.

65. Shokri O, Taghilou S, Geravand F, Paezi M, Molaei M, et al. (2009) Factor structure and psychometric properties of the farsi version of the coping inventory for stressful situations (CISS). Advances in Cognitive Science
$10(3): 22-33$

66. Goldberg DP, Hillier VF (1979) A scaled version of the General Health Questionnaire. Psychological Medicine 9: 139-145.

67. Taghavi MR (2002) Validity ane Reliability of the General Health Questionnaire (GHQ-28) in College Students of Shiraz University. The Journal of Psychology Interdisciplinary and Applied 5(4(20)): 381-398.

68. Feldman G, Greeson J, Senville J (2010) Differential effects of mindful breathing, progressive muscle relaxation, and loving kindness meditation on decentering and negative reactions to repetitive thoughts. Behav Res Ther 48(10): 1002-1011.

69. Lehrer PL, Woolfolk RL, Sime WE (2007) Principles and Practice of Stress Management, $3^{\text {rd }}$ (Edn.), The Guilford Press. United States America.

70. Ranucci C, Pippi R, Buratta L, Aiello C, Gianfredi V, et al. (2017) Effects of an Intensive Lifestyle Intervention to Treat Overweight/Obese Children and Adolescents. Biomed Res Int 2017(5): 1-11.

71. Elvsaas IK $\varnothing$, Giske L, Fure B, Juvet LK (2017) Multicomponent Lifestyle Interventions for Treating Overweight and Obesity in Children and Adolescents: A Systematic Review and Meta-Analyses. J Obes.

72. Bukman AJ, Teuscher D, Feskens EJM (2014) Perceptions on healthy eating, physical activity and lifestyle advice: opportunities for adapting lifestyle interventions to individuals with low socioeconomic status. BMC Public Health 14: 1036.

73. Jowett M (2000) Safe motherhood interventions in low-income countries: an economic justification and evidence of cost effectiveness. Health Policy 53(3): 201-228.

74. Sarris J, O’Neil A, Coulson CE, Schweitzer I, Berk M (2014) Lifestyle medicine for depression. BMC Psychiatry 14: 107.

75. Long SJ, Benton D (2013) Effects of Vitamin and Mineral Supplementation on Stress, Mild Psychiatric Symptoms, and Mood in Nonclinical Samples: A Meta-Analysis. Psychosomatic Medicine 75(2): 144-153.

76. Davison KM, Kaplan BJ (2012) Nutrient intakes are correlated with overall psychiatric functioning in adults with mood disorders. Canadian Journal of Psychiatry 57(2): 85-92.

77. Boffetta P, Couto E, Wichmann J (2010) Fruit and vegetable intake and overall cancer risk in the European Prospective Investigation into Cancer and Nutrition (EPIC). J Natl Cancer Inst 102(8): 529-537.

78. Garcia P, McCarthy M (2000) Measuring Health, A step in the development of city health profiles. World health Organization, Regional Office for Europe Copenhagen.

79. Frank D (2009) Reducing Stress in Adolescent Girls through Outdoor Experiential Education. For degree of Master in Prescott College.

80. Mishara BL, Ystgaard M (2006) Effectiveness of a mental health promotion program to improve coping skills in young children: Zippy's Friends. Early Childhood Research Quarterly 21(1): 110-123.

81. Scheewe TW, Backx FJG, Takken T, Jörg F, van Strater, et al. (2013) Exercise therapy improves mental and physical health in schizophrenia: a randomised controlled trial. Acta Psychiatrica Scandinavica 127(6): 464-473.

82. O’Neil A, Berk M, Itsiopoulos C, Castle D, Opie R, et al. (2013) A randomised, controlled trial of a dietary intervention for adults with major depression (the "SMILES" trial): study protocol. BMC Psychiatry 13: 114 .

83. Kulkarni A, Swinburn B, Utter J (2015) Associations between diet quality and mental health in socially disadvantaged New Zealand adolescents. Eur J Clin Nutr 69(1): 79-83.

84. Sorensen M, Anderssen S, Hjerman I, Holme I, Ursin H (1999) The effect of exercise and diet on mental health and quality of life in middle-aged individuals with elevated risk factors for cardiovascular disease. J sport Sci 17(5): 369-377. 
ISSN: 2574-1241

DOI: $10.26717 /$ BJSTR.2021.36.005787

Esra Tajik. Biomed J Sci \& Tech Res

(C) (P) This work is licensed under Creative

Submission Link: https://biomedres.us/submit-manuscript.php

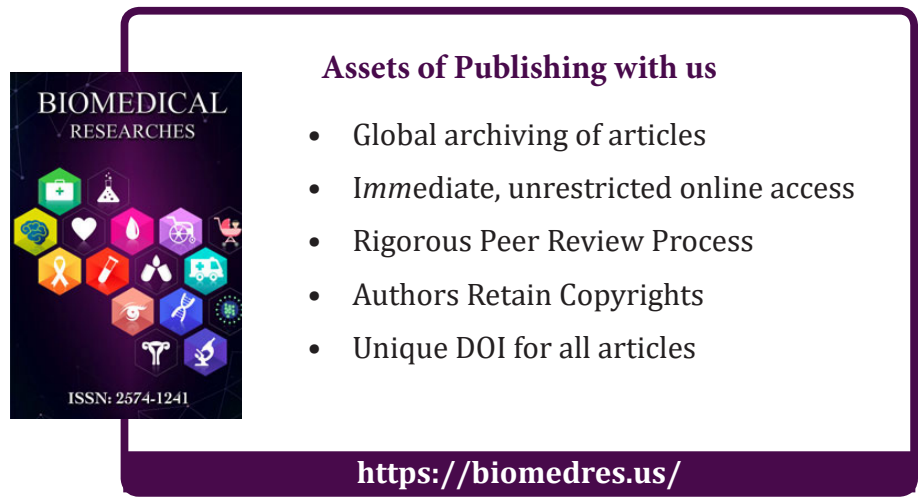

